\title{
DECENTRALIZED POWER ALLOCATION FOR COORDINATED MULTIPLE ACCESS CHANNELS
}

\author{
Elena Veronica Belmega, Samson Lasaulce \\ CNRS - SUPELEC - Univ. Paris Sud \\ 91190 Gif-sur-Yvette, France \\ \{belmega,lasaulce\}@1ss.supelec.fr
}

\author{
Mérouane Debbah \\ Chaire Alcatel-Lucent \\ SUPELEC \\ merouane.debbah@supelec.fr
}

\begin{abstract}
In this paper, a game theoretic approach is used to derive the optimal power allocation in multiple access channels (MAC) for decentralized systems. Unlike previous results, a simple coordination mechanism is used between selfish users. The nature and influence of the coordination mechanism is studied in details for single antenna and multiple antenna terminals. In the proposed framework, the coordination signal indicates to the users in which order the receiver applies successive interference cancelation and with which frequency this order is used: it establishes the rule of the game. Remarkably, in Gaussian single input single output MACs, it is shown that, whatever the rule of the game, the selfish behavior of the users leads to a socially efficient network that is to say it is always sum-capacity achieving. However, for fast fading MAC with multi-antenna terminals, there can be a performance gap between the decentralized system and its centralized counterpart. Analytical and simulation results are provided to assess this gap.
\end{abstract}

\section{INTRODUCTION}

In this paper, we consider a special case of decentralized wireless networks, the decentralized multiple access channel (MAC). In this specific context, the MAC consists of a network of several mobile stations (MS) and one fixed base station (BS). The network is said to be decentralized in the sense that the base station does not dictate to the users the information rates and transmit power levels. Hence, each user can choose freely its power allocation policy in order to selfishly maximize a certain individual performance criterion.

The problem of decentralized power allocation in wireless networks is not new and has been properly formalized for the first time in [1]. Interestingly, the problem can be formulated quite naturally as a non-cooperative game with different performance criteria (utilities) such as the carrier to interference ratio [2], aggregate throughput [3] or energy efficiency [4][5]. In this paper, the point of view adopted is close to the one proposed by the authors of [6] for the DSL (digital subscriber lines) systems, [7] for the single input single output (SISO) and the single input multiple output (SIMO) fast fading multiple access channels with CSIR, CSIT (Channel State Information at the Transmitter and Receiver) and [8] for MIMO
(Multiple Input Multiple Output) with CSIR but only channel distribution information at the transmitters (CDIT). More precisely, we assume, as in [7], that the users want to maximize information-theoretic utilities and more precisely their transmission rate. However our approach differs from [7] on several key technical points. In [7] the authors proposed different formulations (in particular the hierarchical and repeated games formulations) to try to obtain achievable rate regions as close as possible to the centralized MAC capacity region. The corresponding formulations and assumptions made, especially that of the knowledge of all the instantaneous channels at each mobile station, is not applicable to large decentralized networks. In this paper, two key assumptions are made in order to further minimize base station signaling towards the mobiles: the base station can only send to the users a simple coordination signal and sufficient training signals for the users to know the statistics of the different channels. If the channels are stationary the training data will be sent once and for all. The assumed coordination signal is simple because it consists in sending periodically the realization of a $K$ !-state random signal, where $K$ is the number of active users.

Additionally to the assumptions just mentioned, we will assume that both the mobile and base stations are equipped with multiple antennas. Therefore we make the same assumptions as [8] where the authors investigated non-cooperative and non-coordinated MIMO MACs when single-user decoding is assumed at the base station. In our framework the main objective is to know how well a non-cooperative but weakly coordinated system performs in terms of overall sumrate w.r.t. its centralized counterpart when successive decoding is used at the base station. In this setting, several interesting questions arise.

- When the user's utility functions are chosen to be their individual transmission rate, is there a Nash equilibrium in the corresponding game and is it unique?

- What is the optimum way of allocating spatially and temporally the transmit power for a selfish user?

- How do choose the coordination signal in order for the network sum-rate to be maximized? 
In Sec. 2, we begin by considering the SISO static multiple access channel, which is a simple case that provides insights on the stated issues. Then, in Sec. 3 we consider a more attractive framework for wireless networks, which is the fast fading MIMO MAC channel. First we outline the general problem which consists in determining for each user, its optimal space-time power distribution. Then we restrict our attention to two important special cases. In the first case, the spatial correlation between the transmit antennas is assumed to be absent. In this case the optimal spatial power allocation is the uniform power allocation, and the time allocation problem is studied. For the second case, we assume that the temporal power allocation is uniform and thus our objective is to derive the best spatial power allocation scheme. Numerical results are provided in Sec. 4. We conclude by several remarks and possible extensions of our work.

Note: for simplicity sake and without loss of generality, we will assume MACs with two users. However, all the results presented in this paper extend to $K$-user MACs, $K \geq$ 3 . In this respect, in some places $K$ will be used instead of $K=2$.

\section{GAUSSIAN SISO MULTIPLE ACCESS CHANNELS}

In this section we consider a simple case of MACs that captures several important aspects of the problem and serves as baseline example. The two links between the two mobile and base stations are assumed to be static, namely additive white Gaussian (AWGN) channels. We also assume terminals with single antennas. The main feature of the system under investigation is that the base station sends a coordination signal to the (two) users. To generate this signal the base station flips a coin and transmits the corresponding bit, which does not always convey one information bit. Indeed, as this coin is not necessarily fair, it provides a degree of freedom for the base station for optimizing the system performance. The realization of the random signal indicates in which order the base station decodes the users with a perfect successive interference canceler. Note that in a real wireless system the frequency to which the coin would be tossed is roughly proportional to the inverse of the channel coherence time. In the special case of the AWGN MAC, the links are static and therefore the coherence time is infinite.

In order to translate mathematically the proposed coordination scheme, let us denote by $S \in \mathcal{S}$ the coordination signal where $\mathcal{S}=\{1,2\}$ is the set of realizations of $S$ and $\operatorname{Pr}[S=1]=p$ (resp. $\operatorname{Pr}[S=2]=1-p$ ) is the probability that user 1 (resp. user 2) is decoded the second and therefore sees no multiple access interference. The signal received by the base station for the realization $s \in\{1,2\}$ of the coordination signal writes as:

$$
Y^{(s)}=\sum_{k=1}^{2} h_{k} X_{k}^{(s)}+Z^{(s)}
$$

where $h_{k}$ the channel gain for user $k, X_{k}^{(s)}$ is the signal transmitted by user $k$ when the realization of the coordination signal is $s$, and for all $s, Z^{(s)} \sim \mathcal{N}\left(0, \sigma^{2}\right)$ is the (stationary) channel noise. Note that the channel gains are assumed to be known both from the transmitters and receiver. As these gain do not vary over time in this section, acquiring the knowledge of them is not a critical issue here. This can be done once and for all with an arbitrary small loss for the average transmission rate. The chosen transmit power constraints are as follows:

$$
\forall k \in\{1,2\}, p E\left|X_{k}^{(1)}\right|^{2}+\bar{p} E\left|X_{k}^{(2)}\right|^{2} \leq P_{k}
$$

We will use the following notations: $p_{1}=p, \bar{p}_{1}=\bar{p}=$ $1-p, p_{2}=\bar{p}=1-p$ and $\bar{p}_{2}=p$. Also, let $p_{k} \alpha_{k}$ denote the fraction that user $k$ dedicates to the situation where he sees an interference-free channel i.e. $E\left|X_{1}^{(1)}\right|^{2}=\alpha_{1} P_{1}$ and $E\left|X_{2}^{(2)}\right|^{2}=\alpha_{2} P_{2}$. Thus the strategy of a user consists in choosing $\alpha_{k}$ in order to maximize its utility which is his individual average transmission rate. The utility function for user $k \in\{1,2\}$ is given by:

$u_{k}\left(\alpha_{k}, \alpha_{-k}\right)=p_{k} \log \left(1+\alpha_{k} \eta_{k}\right)+\bar{p}_{k} \log \left[1+\frac{\left(1-\alpha_{k} p_{k}\right) \eta_{k}}{\bar{p}_{k}\left(1+\eta_{-k} \alpha_{-k}\right)}\right]$

where the notation $a_{-k}$ stands for the value of the quantity $a$ for the other user and $\eta_{k}=\frac{\left|h_{k}\right|^{2} P_{k}}{\sigma^{2}}$. Given the fact that each user wants to maximize its own utility $u_{k}$ w.r.t. $\alpha_{k} \in\left[0, \frac{1}{p_{k}}\right]$, the main issue is to know if there exists an equilibrium (i.e. no user has interest in deviating from this point), if it is unique and how to determine the corresponding pair of parameters $\left(\alpha_{1}^{*}, \alpha_{2}^{*}\right)$. In fact, the existence and uniqueness of a Nash equilibrium (NE) issues can be dealt with by applying Theorem 1 and 2 of [9] in our context.

Existence of a Nash equilibrium. The existence is insured because of the geometrical and topological properties of the functions $u_{k}$, and of the set over which the maximization is performed. For each user $k \in\{1,2\}$, the function $u_{k}$ is (strictly) concave in $\alpha_{k}$ and continuous in $\left(\alpha_{1}, \alpha_{2}\right)$. For each user $k \in\{1,2\}$, the set of feasible actions or strategies $\mathcal{A}_{k}=\left[0, \frac{1}{p_{k}}\right]$ is convex and compact. The conditions of [9] for the existence of a NE are therefore met.

Uniqueness of the Nash equilibrium. Here, our goal is to prove that the diagonally strict concavity condition of [9] is met in our context and therefore there is a unique NE. For all $\left(\alpha_{1}^{\prime}, \alpha_{1}^{\prime \prime}\right) \in \mathcal{A}_{1}^{2}$ and $\left(\alpha_{2}^{\prime}, \alpha_{2}^{\prime \prime}\right) \in \mathcal{A}_{2}^{2}$ such that either $\alpha_{1}^{\prime} \neq \alpha_{1}^{\prime \prime}$ or $\alpha_{2}^{\prime} \neq \alpha_{2}^{\prime \prime}$ we want to prove that:

$$
\begin{aligned}
\mathcal{C}= & \left\{\left(\alpha_{1}^{\prime \prime}-\alpha_{1}^{\prime}\right)\left[\frac{\partial u_{1}}{\partial \alpha_{1}}\left(\alpha_{1}^{\prime}, \alpha_{2}^{\prime}\right)-\frac{\partial u_{1}}{\partial \alpha_{1}}\left(\alpha_{1}^{\prime \prime}, \alpha_{2}^{\prime \prime}\right)\right]\right. \\
& \left.+\left(\alpha_{2}^{\prime \prime}-\alpha_{2}^{\prime}\right)\left[\frac{\partial u_{2}}{\partial \alpha_{2}}\left(\alpha_{1}^{\prime}, \alpha_{2}^{\prime}\right)-\frac{\partial u_{2}}{\partial \alpha_{2}}\left(\alpha_{1}^{\prime \prime}, \alpha_{2}^{\prime \prime}\right)\right]\right\}>0 .
\end{aligned}
$$


By denoting the two terms of each utility function of eq. (3) as $u_{k}=R_{k}^{(1)}+R_{k}^{(2)}$ the strict positivity condition can be rewritten as follows:

$$
\mathcal{C}=p \mathcal{T}_{1}+(1-p) \mathcal{T}_{2}>0
$$

where for all $s \in\{1,2\}$,

$$
\begin{aligned}
\mathcal{T}_{s}= & \left(\alpha_{1}^{\prime \prime}-\alpha_{1}^{\prime}\right)\left[\frac{\partial R_{1}^{(s)}}{\partial \alpha_{1}}\left(\alpha_{1}^{\prime}, \alpha_{2}^{\prime}\right)-\frac{\partial R_{1}^{(s)}}{\partial \alpha_{1}}\left(\alpha_{1}^{\prime \prime}, \alpha_{2}^{\prime \prime}\right)\right] \\
& +\left(\alpha_{2}^{\prime \prime}-\alpha_{2}^{\prime}\right)\left[\frac{\partial R_{2}^{(s)}}{\partial \alpha_{2}}\left(\alpha_{1}^{\prime}, \alpha_{2}^{\prime}\right)-\frac{\partial R_{2}^{(s)}}{\partial \alpha_{2}}\left(\alpha_{1}^{\prime \prime}, \alpha_{2}^{\prime \prime}\right)\right] .
\end{aligned}
$$

The term $\mathcal{T}_{1}$ can be re-expressed and shown to be positive:

$$
\begin{aligned}
& \mathcal{T}_{1}=\left\{\frac{p\left(\alpha_{1}^{\prime \prime}-\alpha_{1}^{\prime}\right)^{2} \eta_{1}^{2}}{\left(1+\alpha_{1}^{\prime} \eta_{1}\right)\left(1+\alpha_{1}^{\prime \prime} \eta_{1}\right)}\right. \\
& \left.+\frac{p(1-p)^{2}\left(\alpha_{2}^{\prime \prime}-\alpha_{2}^{\prime}\right)^{2} \eta_{2}^{2}-\left(\alpha_{1}^{\prime \prime}-\alpha_{1}^{\prime}\right)\left(\alpha_{2}^{\prime \prime}-\alpha_{2}^{\prime}\right) p^{2}(1-p) \eta_{1} \eta_{2}}{\left(p\left(1+\alpha_{1}^{\prime} \eta_{1}\right)+\eta_{2}-\eta_{2}(1-p) \alpha_{2}^{\prime}\right)\left(p\left(1+\alpha_{1}^{\prime \prime} \eta_{1}\right)+\eta_{2}-\eta_{2}(1-p) \alpha_{2}^{\prime \prime}\right)}\right\}_{1}^{\prime} \\
& \geq \frac{p^{3}\left(\alpha_{1}^{\prime \prime}-\alpha_{1}^{\prime}\right)^{2} \eta_{1}^{2}+p(1-p)^{2}\left(\alpha_{2}^{\prime \prime}-\alpha_{2}^{\prime}\right)^{2} \eta_{2}^{2}-p^{2}(1-p)\left(\alpha_{1}^{\prime \prime}-\alpha_{1}^{\prime}\right)\left(\alpha_{2}^{\prime \prime}-\alpha_{2}^{\prime}\right) \eta_{1} \eta_{2}}{\left(p\left(1+\alpha_{1}^{\prime} \eta_{1}\right)+\eta_{2}-\eta_{2}(1-p) \alpha_{2}^{\prime}\right)\left(p\left(1+\alpha_{1}^{\prime \prime} \eta_{1}\right)+\eta_{2}-\eta_{2}(1-p) \alpha_{2}^{\prime \prime}\right)} \\
& >0
\end{aligned}
$$

In the same way, $\mathcal{T}_{2}$ can be shown to be positive, which concludes the proof of the uniqueness of the NE.

Determination of the Nash equilibrium. In order to find the optimal selfish strategies in the sets of actions $\mathcal{A}_{1}$ and $\mathcal{A}_{2}$ we introduce four Lagrange multipliers $\left(\lambda_{1}, \lambda_{2}, \lambda_{3}, \lambda_{4}\right) \in$ $\left[0,+\infty\left[{ }^{4}\right.\right.$ and define the two constrained functions

$\left\{\begin{array}{l}\mathcal{L}_{1}\left(\alpha_{1}, \alpha_{2}, \lambda_{1}\right)=-u_{1}\left(\alpha_{1}, \alpha_{2}\right)+\lambda_{1}\left(\alpha_{1}-\frac{1}{p}\right)-\lambda_{2} \alpha_{1} \\ \mathcal{L}_{2}\left(\alpha_{1}, \alpha_{2}, \lambda_{2}\right)=-u_{2}\left(\alpha_{1}, \alpha_{2}\right)+\lambda_{3}\left(\alpha_{2}-\frac{1}{1-p}\right)-\lambda_{4} \alpha_{2} .\end{array}\right.$

The Kuhn-Tucker (KT) conditions write as:

$$
\left\{\begin{array}{cl}
\lambda_{1}\left(\alpha_{1}^{*}-\frac{1}{p}\right) & =0 \\
\lambda_{2} \alpha_{1}^{*} & =0 \\
\lambda_{3}\left(\alpha_{2}^{*}-\frac{1}{1-p}\right) & =0 \\
\lambda_{4} \alpha_{2}^{*} & =0
\end{array}\right.
$$

By setting the derivative of the constrained utility functions to zero (8) and using the KT conditions (9) one can check that the pair of parameters at the Nash equilibrium is given by:

$$
\left(\alpha_{1}^{*}, \alpha_{2}^{*}\right)=\mid \begin{array}{cc}
\left(\frac{1}{p}, \frac{1}{1-p}\right) & \text { if } \frac{\eta_{2}}{\eta_{1}}=\frac{1-p}{p} \\
\left(\frac{1}{p}, 1+\frac{\eta_{1}}{\eta_{2}}\right) & \text { if } \frac{\eta_{2}}{\eta_{1}}>\frac{1-p}{p} \\
\left(1+\frac{\eta_{2}}{\eta_{1}}, \frac{1}{1-p}\right) & \text { if } \frac{\eta_{2}}{\eta_{1}}<\frac{1-p}{p} .
\end{array}
$$

Interestingly, we observe that for a fixed game rule, which is the value of parameter $p$, there will always be an equilibrium. The users adapt their strategies to the rule of the game accordingly to eq. (10) in order to optimize their individual transmission rates (3). The base station can therefore choose $p$ to optimize the overall network performance:

$$
p^{*}=\arg \max _{p} R_{\text {sum }}(p)
$$

where $R_{\text {sum }}(p)=u_{1}\left[\alpha_{1}^{*}(p), \alpha_{2}^{*}(p)\right]+u_{2}\left[\alpha_{1}^{*}(p), \alpha_{2}^{*}(p)\right]$. It can be checked that for the tree regimes we defined from the ratio $\frac{\eta_{2}}{\eta_{1}}$ (see eq. (10)), at the equilibrium, the sum-rate is a constant and $R_{\text {sum }}(p)=\log \left(1+\eta_{1}+\eta_{2}\right)$. In fact, this constant is the sum-capacity of the centralized Gaussian SISO MAC. This shows that for any rule of the game, even if it is unfair, the selfish behavior of the users will always lead to maximizing the network sum-rate. Of course, the base station can always chose $p=\frac{1}{2}$ (fair coin) in order to make the game fair without affecting the network performance in the case of Gaussian SISO multiples access channels. However, in general, the proven result means that any binary coordination signal can be used (1-bit quantization of an FM signal, pseudo-random noise generator, etc.) without loss of global optimality.

One can easily check that varying $p$ from 0 to 1 allows one to move along the sum-rate (or full cooperation) segment of centralized Gaussian MAC capacity region, which is a pentagon. The main question that arises is to know to what extent the obtained results apply to the fading MIMO MAC. Indeed, it is known that for the centralized fading MIMO channels, there is generally only one point where the sum-capacity is achieved and therefore there must be some differences to be identified. This is the purpose of the next section.

\section{LARGE FADING MIMO MULTIPLE ACCESS CHANNELS}

\subsection{System Model}

Notations: The notations $\underline{v}$ and $\mathbf{M}$ will stand for vector and matrix respectively. The superscripts $(.)^{T}$ and $(.)^{H}$ will denote transpose and transpose conjugate, respectively. The trace of the matrix $\mathbf{M}$ will be denoted by $\operatorname{Tr}(\mathbf{M})$.

Now each mobile station is equipped with $n_{t}$ antennas whereas the base station has $n_{r}$ antennas (thus we assume the same number of transmitting antennas for all the users). In our analysis the flat fading channel matrices of the different links vary from symbol vector (or space-time codeword) to symbol vector. We assume that the receiver knows all the channel matrices whereas each transmitter has only access to the statistics of the different channels. The equivalent baseband signal received by the base station can be written as

$$
\underline{y}^{(s)}(\tau)=\sum_{k=1}^{K} \boldsymbol{H}_{k}(\tau) \underline{x}_{k}^{(s)}(\tau)+\underline{z}^{(s)}(\tau)
$$

where $\underline{x}_{k}^{(s)}(\tau)$ is the $n_{t}$-dimensional column vector of symbols transmitted by user $k$ at time $\tau$ for the realization $s$ of the coordination signal, $\mathbf{H}_{k}(\tau) \in \mathbb{C}^{n_{r} \times n_{t}}$ is the channel matrix (stationary and ergodic process) of user $k$ and $\underline{z}^{(s)}(\tau)$ is a $n_{r}$-dimensional complex white Gaussian noise distributed as $\mathcal{N}\left(\underline{0}, \sigma^{2} \mathbf{I}_{n_{r}}\right)$. For sake of clarity we will omit the time in$\operatorname{dex} \tau$ from our notations. In order to take into account the 
antenna correlation effects at the transmitters and receiver we will assume the different channel matrices to be structured according to the Kronecker propagation model [10]:

$$
\forall k \in\{1, \ldots, K\}, \mathbf{H}_{k}=\mathbf{R}^{\frac{1}{2}} \Theta_{k} \mathbf{T}_{k}^{\frac{1}{2}}
$$

where $\mathbf{R}$ is the receive antenna correlation matrix, $\mathbf{T}_{k}$ is the transmit antenna correlation matrix for user $k$ and $\boldsymbol{\Theta}_{k}$ is an $n_{r} \times n_{t}$ matrix whose entries are zero-mean independent and identically distributed complex Gaussian random variables with variance $\frac{1}{n_{t}}$.

\subsection{Space-time power allocation game}

Now, in the vector case, the strategy of user $k \in\{1,2\}$, consists in choosing the best pair of precoding matrices $\mathbf{Q}_{k}^{(s)}=$ $E\left[\underline{x}_{k}^{(s)} \underline{x}_{k}^{(s), H}\right]$, for $s \in\{1,2\}$, in the sense of his utility function:

$$
\begin{aligned}
u_{1}\left(\mathbf{Q}_{1}^{(1)}, \mathbf{Q}_{1}^{(2)} \mathbf{Q}_{2}^{(1)}, \mathbf{Q}_{2}^{(2)}\right)= & p R_{1}^{(1)}\left(\mathbf{Q}_{1}^{(1)}, \mathbf{Q}_{2}^{(1)}\right) \\
& +(1-p) R_{1}^{(2)}\left(\mathbf{Q}_{1}^{(2)}, \mathbf{Q}_{2}^{(2)}\right) \\
u_{2}\left(\mathbf{Q}_{1}^{(1)}, \mathbf{Q}_{1}^{(2)} \mathbf{Q}_{2}^{(1)}, \mathbf{Q}_{2}^{(2)}\right)= & p R_{2}^{(1)}\left(\mathbf{Q}_{1}^{(1)}, \mathbf{Q}_{2}^{(1)}\right) \\
& +(1-p) R_{2}^{(2)}\left(\mathbf{Q}_{1}^{(2)}, \mathbf{Q}_{2}^{(2)}\right)
\end{aligned}
$$

where

$$
\begin{aligned}
R_{1}^{(1)}\left(\mathbf{Q}_{1}^{(1)}, \mathbf{Q}_{2}^{(1)}\right)= & \mathbb{E} \log \left|\mathbf{I}+\rho \mathbf{H}_{1} \mathbf{Q}_{1}^{(1)} \mathbf{H}_{1}^{H}\right| \\
R_{2}^{(1)}\left(\mathbf{Q}_{1}^{(1)}, \mathbf{Q}_{2}^{(1)}\right)= & \mathbb{E} \log \left|\mathbf{I}+\rho \mathbf{H}_{1} \mathbf{Q}_{1}^{(1)} \mathbf{H}_{1}^{H}+\rho \mathbf{H}_{2} \mathbf{Q}_{2}^{(1)} \mathbf{H}_{2}^{H}\right| \\
& -\mathbb{E} \log \left|\mathbf{I}+\rho \mathbf{H}_{1} \mathbf{Q}_{1}^{(1)} \mathbf{H}_{1}^{H}\right| \\
R_{1}^{(2)}\left(\mathbf{Q}_{1}^{(2)}, \mathbf{Q}_{2}^{(2)}\right)= & \mathbb{E} \log \left|\mathbf{I}+\rho \mathbf{H}_{1} \mathbf{Q}_{1}^{(2)} \mathbf{H}_{1}^{H}+\rho \mathbf{H}_{2} \mathbf{Q}_{2}^{(2)} \mathbf{H}_{2}^{H}\right| \\
& -\mathbb{E} \log \left|\mathbf{I}+\rho \mathbf{H}_{2} \mathbf{Q}_{2}^{(2)} \mathbf{H}_{2}^{H}\right| \\
R_{2}^{(2)}\left(\mathbf{Q}_{1}^{(2)}, \mathbf{Q}_{2}^{(2)}\right)= & \mathbb{E} \log \left|\mathbf{I}+\rho \mathbf{H}_{2} \mathbf{Q}_{2}^{(2)} \mathbf{H}_{2}^{H}\right|
\end{aligned}
$$

with $\rho=\frac{1}{\sigma^{2}}$ The main point to mention here is the power constraint under which the utilities are maximized. The vector version of the power constraint given in eq. (2) is:

$$
\forall k \in\{1,2\}, p \operatorname{Tr}\left(\mathbf{Q}_{k}^{(1)}\right)+\bar{p} \operatorname{Tr}\left(\mathbf{Q}_{k}^{(2)}\right) \leq n_{t} P_{k}
$$

Obviously the optimum precoding matrices $\mathbf{Q}_{k}^{(s, *)}$ (with $(k, s) \in$ $\{1,2\}^{2}$ ) will depend on $p$. The problem of finding the corresponding matrices $\mathbf{Q}_{k}^{(s, *)}(p)$ and then optimizing the system sum-rate w.r.t. $p$ is doable but requires more space to be treated properly. In this paper we will restrict our attention to two special but interesting cases:

1. Assumption 1. We assume the absence of spatial correlation between the transmit antennas i.e. $\forall k \in\{1,2\}$, $\mathbf{T}_{k}=\mathbf{I}$. Under this assumption, using the fact that the payoff functions are strictly concave, and extending the results given in [11] for the single user channel, we obtain that the optimum precoding matrices are proportional to the $n_{t}$-dimensional identity matrix (uniform spatial allocation), which allows us to convert the space-time power allocation problem into a purely temporal allocation problem (Sec. 3.3).
2. Assumption 2. Here we assume that the user are free to share their transmit power between their antennas but for each realization of the coordination signal the transmit power is constrained as follows (uniform time power allocation):

$$
\forall k \in\{1,2\}, \forall s \in\{1,2\}, \operatorname{Tr}\left(\mathbf{Q}_{k}^{(s)}\right) \leq n_{t} P_{k} .
$$

This is a pure spatial power allocation problem (Sec. 3.4 ).

\subsection{Temporal power allocation game}

Under Assumption 1, the power constraint (16) becomes:

$$
p \alpha_{k}^{(1)}+\bar{p} \alpha_{k}^{(2)} \leq 1,
$$

which is exactly the power constraint we had for Gaussian SISO channels (see eq. (2)). We will use the same notations as in Sec. 2 (e.g. $\alpha_{1}^{(1)}=\alpha_{1}$ ). From now on, we can investigate the same issues as in Sec. 2: the existence of a NE, its uniqueness, its determination and its social efficiency. Because of the lack of space we will not provide all the details of the proofs but only a sketch of them.

Existence of a Nash equilibrium. We apply [9] in our matrix case. Without loss of generality, let us consider user 1. The utility of user 1 comprises two terms corresponding to the two coordination signal realizations: $u_{1}\left(\alpha_{1}, \alpha_{2}\right)=$ $p_{1} R_{1}^{(1)}+\bar{p}_{1} R_{1}^{(2)}$. One can check that:

$$
\begin{aligned}
& \frac{\partial^{2} R_{1}^{(1)}}{\partial \alpha_{1}^{2}}\left(\alpha_{1}, \alpha_{2}\right)=-\mathbb{E} \operatorname{Tr}\left[\mathbf{B B}^{H}\right]<0 \\
& \frac{\partial^{2} R_{1}^{(2)}}{\partial \alpha_{1}^{2}}\left(\alpha_{1}, \alpha_{2}\right)=-\mathbb{E} \operatorname{Tr}\left[\mathbf{C} \mathbf{C}^{H}\right]<0
\end{aligned}
$$

where

$$
\begin{aligned}
\mathbf{B} & =\rho_{1} \mathbf{H}_{1}^{H}\left(\mathbf{I}+\rho_{1} \alpha_{1} \mathbf{H}_{1} \mathbf{H}_{1}^{H}\right)^{-1} \mathbf{H}_{1} \\
\mathbf{C} & =\frac{p}{1-p} \rho_{1} \mathbf{H}_{1}^{H}\left(\mathbf{I}+\rho_{1} \frac{1-p \alpha_{1}}{1-p} \mathbf{H}_{1} \mathbf{H}_{1}^{H}+\rho_{2} \alpha_{2} \mathbf{H}_{2} \mathbf{H}_{2}^{H}\right)^{-1} \mathbf{H}_{1}
\end{aligned}
$$

with $\rho_{k} \triangleq \frac{P_{k}}{\sigma^{2}}$. Thus for each $k$, the utility $u_{k}$ is strictly concave w.r.t. to $\alpha_{k}$. Also it is continuous in $\left(\alpha_{1}, \alpha_{2}\right)$ over the convex and compact sets $\mathcal{A}_{k}=\left[1, \frac{1}{p_{k}}\right]$. Therefore the existence of a NE is guaranteed.

Uniqueness of the Nash equilibrium. We always apply [9] in our matrix case and prove that the diagonally strict concavity condition is always met. The key of the proof is the following Lemma which will not be proved here.

Lemma 3.1 Let $\mathbf{A}^{\prime}, \mathbf{A}^{\prime \prime}, \mathbf{B}^{\prime}$ and $\mathbf{B}^{\prime \prime}$ be Hermitian and positive definite matrices such that either $\mathbf{A}^{\prime} \neq \mathbf{A}^{\prime \prime}$ or $\mathbf{B}^{\prime} \neq \mathbf{B}^{\prime \prime}$. Then we have $\operatorname{Tr}(\mathbf{M}+\mathbf{N})>0$ where

$$
\begin{aligned}
& \mathbf{M}=\left(\mathbf{A}^{\prime \prime}-\mathbf{A}^{\prime}\right)\left[\left(\mathbf{I}+\mathbf{A}^{\prime}\right)^{-1}-\left(\mathbf{I}+\mathbf{A}^{\prime \prime}\right)^{-1}\right] \\
& \mathbf{N}=\left(\mathbf{B}^{\prime}-\mathbf{B}^{\prime \prime}\right)\left[\left(\mathbf{I}+\mathbf{B}^{\prime \prime}+\mathbf{A}^{\prime \prime}\right)^{-1}-\left(\mathbf{I}+\mathbf{B}^{\prime}+\mathbf{A}^{\prime}\right)^{-1}\right]
\end{aligned}
$$


The uniqueness of the NE can then be proved by following the same steps as in the SISO case. The diagonally strict concavity condition can exactly be written as equation (5) but in the matrix case $\mathcal{T}_{1}$ can be checked to be equal to $\operatorname{Tr}(\mathbf{M}+\mathbf{N})$ with

$$
\begin{aligned}
\mathbf{M} & =\left(\mathbf{A}^{\prime \prime}-\mathbf{A}^{\prime}\right)\left[\left(\mathbf{I}+\mathbf{A}^{\prime}\right)^{-1}-\left(\mathbf{I}+\mathbf{A}^{\prime \prime}\right)^{-1}\right] \\
\mathbf{N} & =\left(\mathbf{B}^{\prime}-\mathbf{B}^{\prime \prime}\right)\left[\left(\mathbf{I}+\mathbf{B}^{\prime \prime}+\mathbf{A}^{\prime \prime}\right)^{-1}-\left(\mathbf{I}+\mathbf{B}^{\prime}+\mathbf{A}^{\prime}\right)^{-1}\right] \\
\mathbf{A}^{\prime} & =\rho_{1} \alpha_{1}^{\prime} \mathbf{H}_{1} \mathbf{H}_{1}^{H} \\
\mathbf{A}^{\prime \prime} & =\rho_{1} \alpha_{1}^{\prime \prime} \mathbf{H}_{1} \mathbf{H}_{1}^{H} \\
\mathbf{B}^{\prime} & =\rho_{2} \frac{1-(1-p) \alpha_{2}^{\prime}}{p} \mathbf{H}_{2} \mathbf{H}_{2}^{H} \\
\mathbf{B}^{\prime \prime} & =\rho_{2} \frac{1-(1-p) \alpha_{2}^{\prime \prime}}{p} \mathbf{H}_{2} \mathbf{H}_{2}^{H} .
\end{aligned}
$$

By using Lemma 3.1 twice, one can prove that $\mathcal{T}_{1}$ and $\mathcal{T}_{2}$ are positive, which ensures the unconditional uniqueness of the $\mathrm{NE}$ in the considered game.

Determination of the Nash equilibrium. In order to obtain the NE, we exploit a large system approach, derived in [12] for the fading single user channels. This will lead us to simple approximations of the utility functions which are easier to optimize. From now on we assume the asymptotic regime in terms of the number of antennas: $n_{t} \longrightarrow \infty, n_{r} \longrightarrow \infty$, and $\lim _{n_{t} \rightarrow \infty, n_{r} \rightarrow \infty} \frac{n_{t}}{n_{r}}=c<\infty$. Under these assumptions, for $s=1$ (the expressions for $s=2$ can be written in a similar way) and each $k \in\{1,2\}$, the rates $R_{k}^{1)}$ can be can be shown to be equivalent to [12]:

$$
\begin{aligned}
\tilde{R}_{1}^{(1)}= & n_{t} \log _{2}\left(1+\rho_{1} \alpha_{1} \gamma_{1}\right)+ \\
& +n_{r} \log _{2}\left(c \gamma_{1}\right)-n_{t} \gamma_{1}\left(c \gamma_{1}-1\right) \log _{2} e \\
\tilde{R}_{2}^{(1)}= & n_{t} \log _{2}\left(1+\rho_{1} \alpha_{1} \gamma_{1}\right)+ \\
& +n_{t} \log _{2}\left[1+2 \rho_{2} \frac{1-(1-p) \alpha_{2}}{p} \gamma_{2}\right]+ \\
& +n_{r} \log _{2}\left(2 c \gamma_{2}\right)-4 n_{t} \gamma_{2}\left(c \gamma_{2}-1\right) \log _{2} e \\
& -\tilde{R}_{1}^{(1)}
\end{aligned}
$$

where the parameters $\gamma_{j}, j \in\{1,2\}$ are solutions of the following 2-degree equations:

$$
\begin{aligned}
\gamma_{1} & =\frac{n_{r}}{n_{t}} \frac{1}{1+\rho \frac{\alpha_{1} P_{1}}{1+\rho \alpha_{1} P_{1} \gamma_{1}}} \\
\gamma_{2} & =\frac{n_{r}}{n_{t}} \frac{1}{+\rho\left(\frac{\alpha_{1} P_{1}}{1+2 \rho \alpha_{1} P_{1} \gamma_{2}}+\frac{\left(1-(1-p) \alpha_{2}\right) P 2}{p+2 \rho\left(1-(1-p) \alpha_{2}\right) P 2 \gamma_{2}}\right)}
\end{aligned}
$$

The approximates of $\tilde{R}_{2}^{(1)}$ and $\tilde{R}_{1}^{(2)}$ can be obtained in a similar way and the approximated utilities follows since:

$$
\begin{aligned}
& \tilde{u}_{1}\left(\alpha_{1}, \alpha_{2}\right)=p \tilde{R}_{1}^{(1)}\left(\alpha_{1}, \alpha_{2}\right)+(1-p) \tilde{R}_{1}^{(2)}\left(\alpha_{1}, \alpha_{2}\right) \\
& \tilde{u}_{2}\left(\alpha_{1}, \alpha_{2}\right)=p \tilde{R}_{2}^{(1)}\left(\alpha_{1}, \alpha_{2}\right)+(1-p) \tilde{R}_{2}^{(2)}\left(\alpha_{1}, \alpha_{2}\right) .
\end{aligned}
$$

Now in order to solve the constrained optimization problem, we introduce the Lagrange multipliers $\left(\lambda_{1}, \lambda_{2}, \lambda_{3}, \lambda_{4}\right) \in[0,+\infty$ and define the two functions:

$$
\begin{aligned}
& \mathcal{L}_{1}\left(\alpha_{1}, \alpha_{2}, \lambda_{1}\right)=-\tilde{u}_{1}\left(\alpha_{1}, \alpha_{2}\right)+\lambda_{1}\left(\alpha_{1}-\frac{1}{p}\right)-\lambda_{2} \alpha_{1} \\
& \mathcal{L}_{2}\left(\alpha_{1}, \alpha_{2}, \lambda_{2}\right)=-\tilde{u}_{2}\left(\alpha_{1}, \alpha_{2}\right)+\lambda_{3}\left(\alpha_{2}-\frac{1}{1-p}\right)-\lambda_{4} \alpha_{2} .
\end{aligned}
$$

The Kuhn-Tucker (KT) conditions can be written in the same way as in (9). Therefore the optimum selfish power allocations can be obtained by using a fixed-point method and an iterative algorithm following the same idea as in [8] for noncoordinated MIMO MACs with single-user decoding. At this point we have to make an important technical comment. Our proof for the existence and uniqueness of the NE holds for the exact game. For the approximated game, we need the approximated utilities to have the same properties as their exact counterparts. It turns out that the large system approximation of the ergodic mutual information can be shown to have the desired properties [13].

Now, let us focus on the network sum-rate. As the optimum precoding matrices are proportional to the identity matrix, it can be checked that the sum-capacity of the considered decentralized MAC is reached for $p=0$ and $p=1$ : $R_{\text {sum }}(p)=\mathbb{E} \log \left|\mathbf{I}+\rho_{1} \mathbf{H}_{1} \mathbf{H}_{1}^{H}+\rho_{2} \mathbf{H}_{2} \mathbf{H}_{2}^{H}\right|$. Unlike the SISO case, it is not reached for any value of $p$. In particular, the fair choice $p=\frac{1}{2}$ does not lead to a decentralized network achieving the same sum-capacity of its centralized counterpart. We will quantify the corresponding suboptimality through simulation results.

\subsection{Spatial power allocation game}

In this section we assume that the user are free to share their transmit power between their antennas but for each realization of the coordination signal the transmit power is constrained as follows: $\forall k \in\{1,2\}, \forall s \in\{1,2\}, \operatorname{Tr}\left(\mathbf{Q}_{k}^{(s)}\right) \leq P_{k}$. This means that we assume that the users cannot distribute their power over time: they cannot decide the amount of power they dedicate to a given realization of the coordination signal. The main feature of the game under the aforementioned power constraint is that there exists a unique Nash equilibrium in each sub-game defined by the realization of the coordination signal. The proof is much simpler than that of the time power allocation problem since the use of Rosen's Theorem [9] is not required. Without loss of generality assume that $S=1$. Whatever the strategy of user 2 , user 1 sees no multiple access interference. Theferore he can choose $\mathbf{Q}_{1}^{(1)}$ independently of user 2 . As $R_{1}^{(1)}\left(\mathbf{Q}_{1}^{(1)}, \mathbf{Q}_{2}^{(1)}\right)$ is a strictly concave function to be maximized over a convex set, there is a unique optimum strategy for user 1 . As we assume a game with complete information, user 2 knows the utility of user 1 and thus the precoding matrix he chooses. The same concavity argument can be used for $R_{2}^{(1)}\left(\mathbf{Q}_{1}^{(1)}, \mathbf{Q}_{2}^{(1)}\right)$ and therefore guarantees that user 2 will use a given precoding matrix.

Determination of the Nash equilibrium. In order to find [the optimum covariance matrices we proceed in the same way as described in [8]. First we will focus on the optimum eigenvectors and then we will determine the optimum eigenvectors by approximating the utility functions under the large system assumption. In order to determine the optimum eigenvectors the proof in [8] can be applied to our case to assert that there is 
no loss of optimality by restricting the search for the optimum covariance matrix by imposing the structure $\mathbf{Q}_{k}=\mathbf{U}_{k} \mathbf{P}_{k} \mathbf{U}_{k}$, where $\mathbf{T}_{k}=\mathbf{U}_{k} \mathbf{D}_{k} \mathbf{U}_{k}$ is the spectral decomposition of the transmit correlation matrix defined in (13) and the diagonal matrix $\mathbf{P}_{k}=\operatorname{Diag}\left(P_{k}(1), \ldots, P_{k}\left(n_{t}\right)\right)$ represents the powers of user $k$ allocated to the different eigenvectors.

As a consequence, we can exploit once again the results of [12], assuming the asymptotic regime in terms of the number of antennas, defined in the previous subsection. The new approximated utilities are:

$$
\begin{aligned}
\tilde{R}_{1}^{(1)}= & \sum_{i=1}^{n_{t}} \log _{2}\left[1+\rho P_{1}^{(1)}(i) d_{1}(i) \gamma_{1}\right]+ \\
& +\sum^{2} j=1^{n_{r}} \log _{2}\left[1+\rho d^{(R)}(j) \delta_{1}\right]-n_{t} \rho \gamma_{1} \delta_{1} \log _{2} e \\
\tilde{R}_{2}^{(1)}= & \sum_{l=1}^{n_{t}} \sum_{i=1}^{n_{r}} \log _{2}\left[1+2 \rho P_{l}^{(1)}(i) d_{l}(i) \gamma_{2}\right]+ \\
& +\sum_{j=1}^{n_{r}} \log _{2}\left[1+2 \rho d^{(R)}(j) \delta_{2}\right]-4 n_{t} \rho \gamma_{2} \delta_{2} \log _{2} e- \\
& -\tilde{R}_{1}^{(1)}
\end{aligned}
$$

where $d_{l}, l \in\{1,2\}$ are the vectors that contain the eigenvalues of the transmit correlation matrices $\mathbf{T}_{l}, d^{(R)}$ contains the eigenvalues of the receive correlation matrix $\mathbf{R}$. Also the coefficients $\gamma_{1}, \delta_{1}, \gamma_{2}, \delta_{2}$ are the solutions of the following systems

$$
\begin{gathered}
\left\{\begin{array}{l}
\gamma_{1}=\frac{1}{n_{t}} \sum_{j=1}^{n_{r}} \frac{d^{(R)}(j)}{1+\rho d^{(R)}(j) \delta_{1}} \\
\delta_{1}=\frac{1}{n_{t}} \sum_{i=1}^{n_{t}} \frac{P_{1}^{(1)}(i) d_{1}(i)}{1+\rho P_{1}^{(1)}(i) d_{1}(i) 1 \gamma_{1}}
\end{array}\right. \\
\left\{\begin{array}{l}
\gamma_{2}=\frac{1}{2 n_{t}} \sum_{j=1}^{n_{r}} \frac{d^{(R)}(j)}{1+2 \rho d^{(R)}(j) \delta_{2}} \\
\delta_{2}=\frac{1}{2 n_{t}} \sum_{l=1}^{2} \sum_{i=1}^{n_{t}} \frac{P_{l}^{(1)}(i) d_{l}(i)}{1+2 \rho P_{l}^{(1)}(i) d_{l}(i) 1 \gamma_{2}}
\end{array}\right.
\end{gathered}
$$

Then, optimizing the approximated rates $\tilde{R}_{1}^{(1)}$ w. r. t. $P_{1}^{(1)}(i)$, and then $\tilde{R}_{2}^{(1)}$ w.r.t. $P_{1}^{(1)}(i)$ leads to the following waterfilling equations:

$$
\begin{aligned}
& P_{1}^{(1), *}(i)=\left[\frac{1}{n_{r} \ln 2 \lambda_{1}}-\frac{1}{\rho d_{1}(i) \gamma_{1}}\right]^{+} \\
& P_{2}^{(1), *}(i)=\left[\frac{1}{n_{r} \ln 2 \lambda_{2}}-\frac{1}{\rho d_{2}(i) \gamma_{2}}\right]^{+}
\end{aligned}
$$

where $\lambda_{k} \geq 0$ and for user $k$ is the Lagrangian multipliers tuned in order to meet the power constraint given in (17): $\sum_{i=1}^{n_{t}} P_{k}^{(1)}(i)=n_{t} P_{k}$. We use the same iterative power allocation algorithm as the one described in [8]. As we have mentioned in the previous subsection, the results of [13] show that the approximated utilities are strictly concave, but also that if the iterative power algorithm converges, it converges towards the global maximum. We obtain in this case also that, under the large systems assumption, the approximated utilities have the same properties as the exact utilities.

One important point to notice here is that the obtained optimum precoding matrices do not depend on $p$. This considerably simplifies the base station's choice for the sum-rate optimal value for $p$. Indeed, the sum-rate $R_{\text {sum }}(p)$ is merely a linear function of $p: R_{\text {sum }}(p)=a p+b$ where

$$
\begin{aligned}
a= & \mathbb{E} \log \left|\mathbf{I}+\rho \mathbf{H}_{1} \mathbf{Q}_{1}^{(1), *} \mathbf{H}_{1}^{H}+\rho \mathbf{H}_{2} \mathbf{Q}_{2}^{(1), *} \mathbf{H}_{2}^{H}\right| \\
& -\mathbb{E} \log \left|\mathbf{I}+\rho \mathbf{H}_{1} \mathbf{Q}_{1}^{(2), *} \mathbf{H}_{1}^{H}+\rho \mathbf{H}_{2} \mathbf{Q}_{2}^{(2), *} \mathbf{H}_{2}^{H}\right| \\
b= & \mathbb{E} \log \left|\mathbf{I}+\rho \mathbf{H}_{1} \mathbf{Q}_{1}^{(2),{ }^{*}} \mathbf{H}_{1}^{H}+\rho \mathbf{H}_{2} \mathbf{Q}_{2}^{(2), *} \mathbf{H}_{2}^{H}\right| .
\end{aligned}
$$

Depending on the sign of $a$, if the base station wants to maximize the sum-rate it will choose $p=0$ or $p=1$. If it wants a fair game it will choose $p=\frac{1}{2}$ and accept a certain loss of global optimality. Note that even for $p \in\{0,1\}$ the sum-capacity is not reached in general: this is because the matrix $\mathbf{Q}_{1}^{(1) \text {,* }}$ (resp. $\mathbf{Q}_{2}^{(2), *}$ ) does not coincide with the first (resp. second) component of the pair of precoding matrices that maximizes the (strictly concave) network sum-rate.

\section{SIMULATION RESULTS}

For the temporal power allocation problem we have seen that the decentralized MAC depends on the rule of the game i.e. the value of $p$. This is exactly what Fig. 1 depicts for the following scenario: $P_{1}=10, P_{2}=1, \rho=5 \mathrm{~dB}, n_{t}=n_{r}=4$. First, we see that the MAC sum-rate is a convex function of $p$ and the maximum of $R_{\text {sum }}(p)$ is reached for $p=0$ and $p=1$. One important observation to be made is that the minimum and maximum only differ by about $1 \%$. Many other simulations have confirmed this observation. This shows that the sub-optimality is not marked. This means that any game rule can lead to a almost sum-rate optimal game, this holds in particular for a fair game. Fig. 2 shows the set of rate pair achieved by varying $p$ from 0 to 1 . The corresponding looks like a straight line and is very close to the sum-rate line given by the equation $R_{1}+R_{2}=C_{\text {sum }}$ where $C_{\text {sum }}$ is the centralized MAC sum-capacity. It is interesting to note that the base station can, through a single parameter (i.e. $p$ ), force the system to operate at different many points which corresponds to relatively large ranges of of transmission rates. It is also very interesting to note, that the fast MIMO MAC behaves like a Gaussian SISO MAC in the sense that the capacity region border comprises a segment over which the sum-rate is reached (up to a small error). This is not true for fast fading SISO MACs. Here, we observe the consequence of the double averaging effect present in the considered utilities (ergodic rates plus spatial averaging). This shows that, although the ergodic capacity region of the centralized MAC with CSIR and CDIT is not known in general, it can be determined up to a small relative error under the large system approximation and therefore make possible the analysis of the decentralized MAC. 
Now we consider the purely spatial power allocation problem. We already know that the sum-rate is a linear function of $p$ and therefore is maximum in $p=0$ or $p=1$. It turns out that this slope has a small value. This observation has been confirmed by many simulation scenarios. This slope is even 0 for a symmetric MAC i.e. $P_{1}=P_{2}$ and $\mathbf{T}_{1}=\mathbf{T}_{2}$. This can be checked analytically by inspecting the sum-rate expression. Fig. 3 shows the set of rate pairs achieved by varying $p$ for the scenario: $P_{1}=10, P_{2}=1, \rho=3 \mathrm{~dB}$, $n_{t}=n_{r}=5, t_{1}=t_{2}=0.2$ where $t_{k}$ is the correlation parameter that characterize the correlation matrix chosen to be given by $T_{k}(i, j)=t_{k}^{i-j \mid}$. Even in this scenario, chosen to be a bad case in terms of sub-optimality, the sum-rate is not far from the sum-capacity of the centralized MAC.

\section{SUMMARY}

In this contribution, we have provided a game-theoretic framework for decentralized multiple access channels using a simple coordination mechanism. We have provided several interesting theoretical and simulation results. First, we proved the existence and uniqueness of a Nash equilibrium both for decentralized Gaussian SISO and fast fading MIMO MACs. We also provided the corresponding optimum selfish power allocation policies. We have seen that the base station can, through a single parameter (i.e. $p \in[0,1]$, which represents the distribution of the coordination signal), force the system to operate at different many points which corresponds to relatively large ranges of of transmission rates. For Gaussian MACs with single antenna terminals, the corresponding set of rates corresponds to the full cooperation segment of the centralized MAC. Said otherwise a decentralized Gaussian SISO MAC with coordination achieves the same rate pairs as a MAC with full cooperation or virtual MIMO system. As a second step we wanted to know to what extent this key result extends to fading MAC with multi-antenna terminals. It turns out this is almost true in the MIMO setting. For the cases of interest where the power was optimally allocated either over space or time, the performance gap is relatively small. Interestingly in large MIMO MACs, the capacity region comprises a full cooperation segment just like SISO MACs. The coordination signal precisely allows one to move along the corresponding (almost) straight line. This shows the relevance of large systems in distributed networks since they allow to determine the capacity region of certain systems whereas it is unkwnown in the finite setting and also induce an averaging effect, which makes the behavior or users predictible. Indeed, in large MIMO MACs the knowledge of the CDIT does not involve any performance w.r.t. the case with perfect CSIT.

\section{REFERENCES}

[1] S. A. Grandhi, R. Vijayan and D. J. Goodman, "Distributed power control in cellular radio systems", IEEE

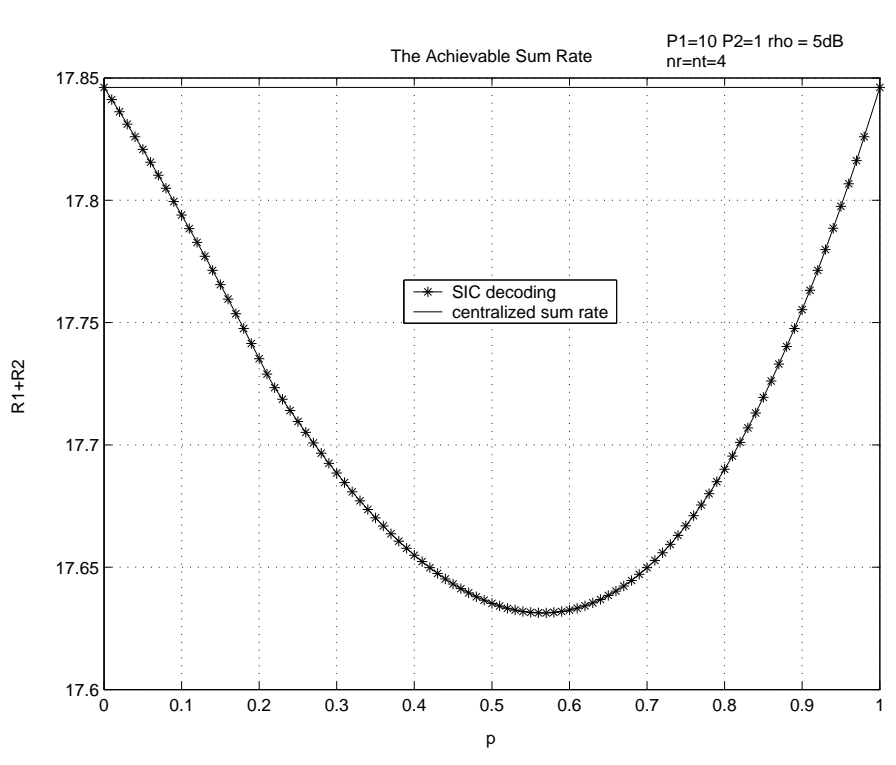

Fig. 1. Temporal power allocation case. MAC sum-rate versus $p$ for $P_{1}=10, P_{2}=1, n_{r}=n_{t}=4, \rho=5 d B$. The sum-rate of fading MIMO MACs is almost reached whatever the coordination signal distribution.

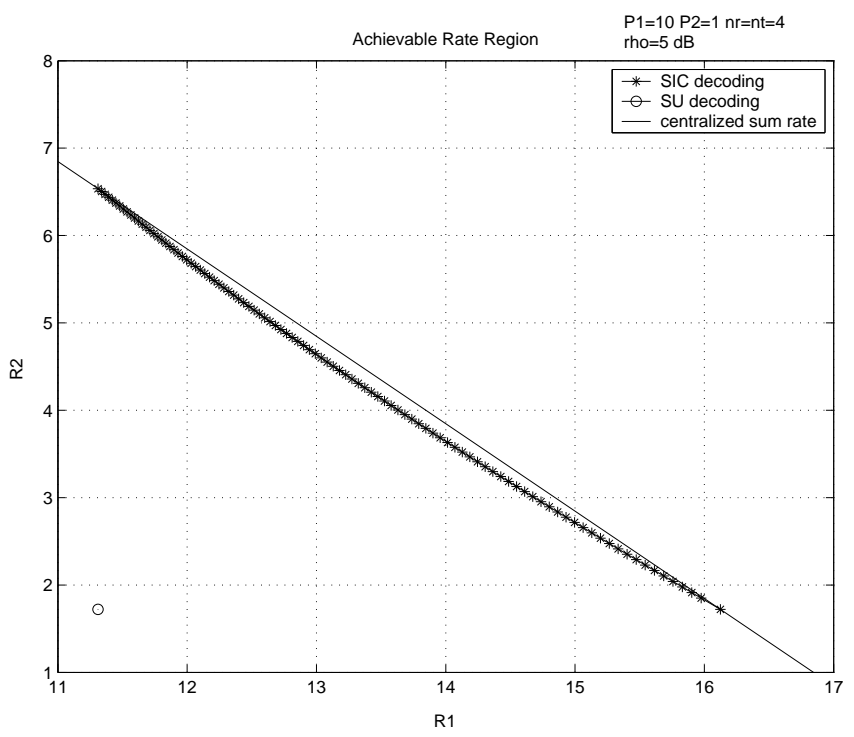

Fig. 2. Achievable rate pairs for the temporal power allocation case for $P_{1}=10, P_{2}=1, n_{r}=n_{t}=4, \rho=5 \mathrm{~dB}$. The performance of the decentralized MAC with coordination is close to that of its centralized counterpart. 


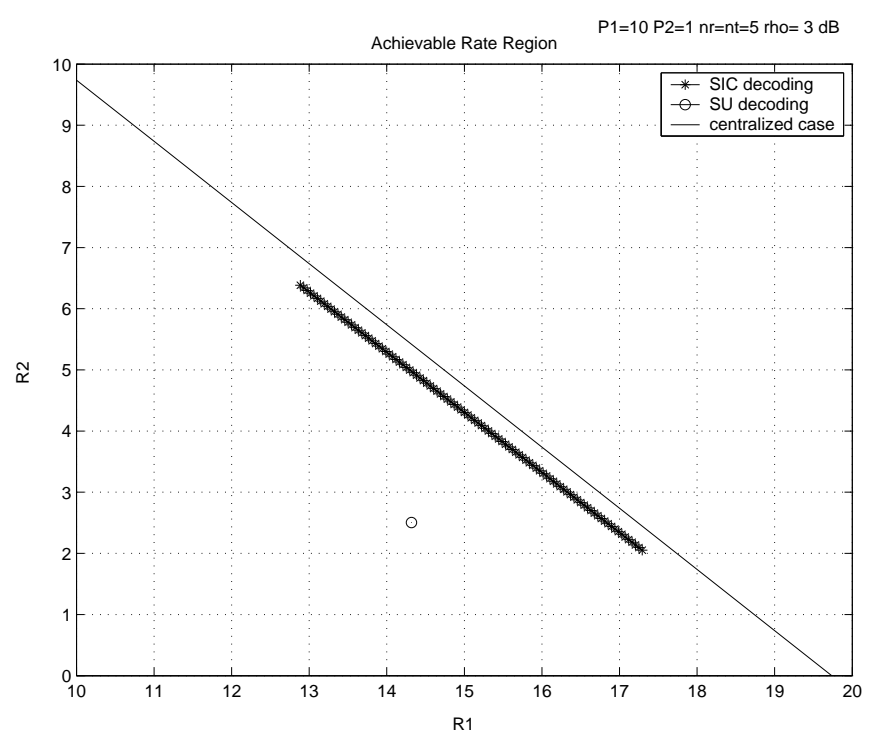

Fig. 3. Achievable rate pairs for the spatial power allocation case for $P_{1}=10, P_{2}=1, n_{r}=n_{t}=5, t_{k}=0.2, \rho=3 \mathrm{~dB}$. The performance of the decentralized MAC with coordination is close to that of its centralized counterpart.

Transactions on Communications, Vol. 42, No. 234, pp. 226-228, Feb/Mar/Apr 1994.

[2] H. Ji and C.-Y. Huang, "Non-cooperative uplink power control in cellular radio systems", Wireless Networks, Vol. 4, No. 3, pp. 233-240, 1998.

[3] S.-J. Oh, T. L. Olsen and K. M. Wasserman, "Distributed power control and spreading gain allocation in CDMA data networks", IEEE Proc. of INFOCOM, Vol. 2, March 2000, pp. 379-385.

[4] D. J. Goodman and N. B. Mandayam, "Power Control for Wireless Data", IEEE Personal Communications, Vol. 7, No. 2, pp. 48-54, April 2000.

[5] F. Meshkati, M. Chiang, H. V. Poor and S. C. Schwartz, "A game-theoretic approach to energy-efficient power control in multi-carrier CDMA systems", IEEE Journal on Selected Areas in Communications, Vol. 24, No. 6, pp. 1115-1129, June 2006.

[6] W. Yu, G. Ginis and J. M. Cioffi, "Distributed multiuser power control for digital subscriber lines"; IEEE Journal of Selected Areas in Communications, Vol. 20, No. 5, June 2002, pp. 1105-1115.

[7] L. Lai and H. El Gamal, "The water-filling game in fading multiple access channels", sub. to IEEE Trans. on Inform. Theory, 2005.
[8] S. Lasaulce, A. Suarez, M. Debbah and L. Cottatellucci, "Power allocation game for fading MIMO multiple access channels with antenna correlation, in the ICST/ACM proc. of the International Conference on Game Theory in Communications Networks (Gamecomm), Nantes, France, Oct. 2007.

[9] J. Rosen, "Existence and uniqueness of equilibrium points for concave n-person games", Econometrica, Vol. 33, pp. 520-534, 1965.

[10] D. S. Shiu, G. J. Foschini, M. J. Gans and J. M. Kahn, "Fading correlation and its effects on the capacity of multielement antenna systems", IEEE Trans. on Comm., Vol. 48, No. 3, Mar. 2000, pp. 502-513.

[11] E. Telatar, "Capacity of Multi-Antenna Gaussian Channels", European Transaction on Telecommunications, Vol. 10, No. 6, Nov. 1999, pp. 585-596.

[12] A. Tulino and S. Verdu, "Random Matrices and Wireless Communications", Foundations and trends in communications and information theory, NOW, The Essence of Knowledge, 2004.

[13] J. Dumont, W. Hachem, S. Lasaulce, P. Loubaton and J. Najim, "On the capacity achieving covariance matrix of Rician MIMO channels: an asymptotic approach", IEEE Transactions on Information Theory, submitted, available on the Arxiv website. 